\title{
Front Matter: Volume 10312
}

, "Front Matter: Volume 10312," Proc. SPIE 10312, Neural and Fuzzy Systems: The Emerging Science of Intelligent Computing, 1031201 (28 June 1994); doi: $10.1117 / 12.2283784$

SPIE Event: SPIE Institutes for Advanced Optical Technologies 12, 1994, SPIE. Bellingham, WA, United States 


\section{CONTENTS}

ix Series Introduction

xi Preface

xiii Contributors

3 Chaos and information loss in fuzzy dynamical systems

P. Diamond, Univ. of Queensland (Australia)

28 Neural network implementation of fuzzy inference for approximate case-based reasoning

L. Ding, Z. Shen, National Univ. of Singapore (Singapore)

57 Synthesis of fuzzy artificial intelligence and neural networks for hierarchical intelligent control

T. Fukuda, T. Shibata, Nagoya Univ. (Japan)

85 Neural learning of robot inverse kinematics transformations

M. M. Gupta, D. H. Rao, Univ. of Saskatchewan (Canada)

113 Tether operations in space using fuzzy-logic-based depth control

R. N. Lea, NASA/Johnson Space Center; Y. Jani, Togai InfraLogic, Inc.

133 Fuzzy and possibilistic clustering methods for computer vision

R. J. Krishnapuram, J. M. Keller, Univ. of Missouri/Columbia

160 Neuro-fuzzy image processing: relevance and feasibility

S. K. Pal, A. Chosh, Indian Statistical Institute (India)

185 Adaptive pattern recognition by self-organizing neural networks

S. Mitra, Y. Kim, S. Pemmaraju, Texas Tech Univ.

215 Fuzzy morphological algorithms

E. R. Dougherty, Rochester Institute of Technology; D. Sinha,

CUNY/College of Staten Island; P. Sinha, Stevens Institute of Technology

264 Analysis and segmentation of higher-dimensional data sets with fuzzy operators for representation and visualization

W. F. Kraske, Northrop Corp.

302 Clustering analysis of multidimensional fuzzy sets using mathematical morphology

A. Kher, S. Mitra, Texas Tech Univ.

Neural and Fuzzy Systems: The Emerging Science of Intelligent Computing, edited by Sunanda D. Mitra, Madan M. Gupta, Wolfgang F. Kraske, Proc. of SPIE Vol. 10312 (Vol. IS12), 1031201 @ (1994) 2017 SPIE · CCC code: 0277-786X/17/\$18 - doi: 10.1117/12.2283784

vii Proc. of SPIE Vol. 10312 1031201-1 


\section{SERIES INTRODUCTION}

Neural and Fuzzy Systems: The Emerging Science of Intelligent Computing, twelfth in the SPIE Institute Series, is representative of SPIE's primary goal for this series: to provide, in a timely manner, authoritative overall introductions and reviews of emerging or critical technologies based on or related to optics and optoelectronics.

The eleven contributions present topics in what one might term the "science of reasoning," discussing, as they do, the principles and algorithms for neural network and autonomous robot systems. Matters of control and decision in systems having nonlinear dynamic characteristics are at the core of modern research in these areas. Again, as demonstrated by previous Institute volumes, the time lapse between fundamental research and technological applications in this field is narrowing significantly. Developments on one side of the R\&D ledger lead to, and are balanced by, new concepts and approaches in the other. The Institute Series hopes to encourage and abet such synergism.

It is expected that this book will be of interest to those bodies of scientists and engineers involved in the R\&D of a variety of technologies-computer vision, image processing, artificial intelligence, neural networks, and robotic command and control. That there are other technologies that might have an interest in and benefit from developments in this field of neural and fuzzy systems, I have no doubt.

\section{Roy F. Potter}

General Editor

SPIE Institutes for Advanced Optical Technologies 


\section{PREFACE}

The concept of adaptive learning of system parameters in nonlinear systems has been addressed by system theorists for quite some time. The independent use of neural networks and fuzzy logic to facilitate such adaptive learning is, however, very recent. An even more recent trend has been to combine the strength of adaptive learning in neural networks with the fuzzy membership values of input/output data for designing better identification, recognition, and control systems.

The idea of publishing an SPIE Institute Series volume on the emerging science of intelligent computing was first discussed with Roy F. Potter, the series editor, while planning a workshop on Neuro-Control Systems that was held during the SPIE Symposium on Aerospace Sensing, 20-24 April 1992 in Orlando. This workshop was made possible by the sponsorship of Northrop Corporation through a symposium grant awarded to Texas Tech University. Keynote speakers at the workshop included Lotfi Zadeh (Univ. of California/Berkeley), James Bezdek (Univ. of West Florida), Madan Gupta (Univ. of Saskatchewan), James Keller (Univ. of Missouri/Columbia), and Robert Lea (NASA/JSC). Wolfgang Kraske (Northrop Corp.) and Sunanda Mitra (Texas Tech Univ.) were among the other speakers.

This twelfth volume in the SPIE Institute Series, Neural and Fuzzy Systems, presents some of the ideas and applications involving neural networks and/or fuzzy logic for better representation and operation of dynamical systems. Eleven chapters from researchers in six countries have been included. Chapter 1 describes the general behavior of nonlinear dynamical systems that are often chaotic. During the evolution of a dynamical system, information content may undergo degradation under iteration of usual fuzzified chaotic mappings. This chapter investigates more general classes of fuzzy chaos based on tnorms/conorms. Chapter 2 discusses the implementation of fuzzy rules for approximate case-based reasoning in neural network units. Chapter 3 presents a hierarchical structure of intelligent servo control for robotic motion, including fuzzy logic combined with neural networks. Chapter 4 describes a neural model for constrained inverse kinematic transformations for two- and three-linked robots. Chapter 5 presents a fuzzy controller model of highly complex and nonlinear dynamics of tether operations in space. Chapters 6 through 8 investigate different approaches for fuzzy and neuro-fuzzy techniques as applied to problems in computer vision, image processing, and pattern recognition. Chapters 9 through 11 discuss fuzzy and fuzzy morphological operators and algorithms used in analyzing higher-dimensional data sets. Chapters 6 and 8 present new neuro-fuzzy and possibilistic approaches to clustering techniques, while Chapter 11 reviews current fuzzy morphological filtering techniques for clustering noisy multidimensional data. 
Clustering is a well-known technique that has been used primarily for partitioning complex data sets of unknown data distribution. However, interest has grown recently in using clustering for identification of system parameters in nonlinear dynamical systems.

I would like to thank Madan Gupta for his encouragement in organizing the workshop in Orlando, as well as the other keynote speakers, Lotfi Zadeh, James Bezdek, James Keller, and Robert Lea, for their valuable help in making the workshop successful. I am grateful also to Wolfgang Kraske, without whose support the workshop and book would not have materialized, and to Roy Potter, editor of the SPIE Institute Series. Finally I would like to thank SPIE staff Mary Horan, Anne Noteboom, and Eric Pepper, and all the authors, for their valuable contributions and efforts in making this volume possible.

\section{Sunanda Mitra}

Texas Tech University

February 1994 


\section{CONTRIBUTORS}

Phil Diamond

University of Queensland

Mathematics Department

Queensland QLD 4072

Australia

Liya Ding

National University of Singapore

Institute of Systems

Heng Mui King Terrance

Kent Ridge 0511

Singapore

Edward R. Dougherty

Rochester Institute of Technology

Center for Imaging Science

One Lomb Dr.

Rochester, NY 14623

Toshio Fukuda

Nagoya University

Department of Mechano-Informatics and Systems

1 Furo-cho, Chikusa-ku

Nagoya 464-01

Japan

Ashish Ghosh

Indian Statistical Institute

Machine Intelligence Unit

203 B. T. Road

Calcutta 700035

India

Madan M. Gupta

University of Saskatchewan

Intelligent Systems

Research Laboratory

College of Engineering

Saskatoon SK S7N OWO

Canada
Yashvant Jani

Togai InfraLogic, Inc.

17000 El Camino Real, Ste.204C

Houston, TX 77058-2636

James M. Keller

University of Missouri/Columbia

Department of Electrical and

Computer Science

217 Electrical Eng. Building

Columbia, MO 65211

Alok Kher

Texas Tech University

Computer Vision and Image

Analysis Laboratory

MS 3102, Department of Electrical

Engineering

P.O. Box 43102

Lubbock, TX 79409-3102

Yong Soo Kim

Texas Tech University

Computer Vision and Image

Analysis Laboratory

Department of Electrical Engineering

P.O. Box MS 3102

Lubbock, TX 79409-3102

Wolfgang F. Kraske

Northrop Corporation

Aircraft Group

B-2 Div., Department of Advanced

Special Projects

8900 E Washington Blvd., W921

Pico Rivera, CA 90660-3765

Raghu J. Krishnapuram

University of Missouri/Columbia

221 EE B ldg., E\&CE Department

Columbia, MO 65211-0001

xiii 
Robert N. Lea

NASA/Johnson Space Center

Software Technology Group, PT4

Houston, TX 77058

Sunanda Mitra

Texas Tech University

Computer Vision and Image

Analysis Laboratory

MS 3102, Department of EE

P.O. Box 43102

Lubbock, TX 79409-3102

Sankar K. Pal

Indian Statistical Institute

Machine Intelligence Unit

203 B. T. Road

Calcutta 700035

India

Suryalakshmi Pemmaraju

Texas Tech University

Computer Vision and Image

Analysis Laboratory

Department of Electrical Engineering

P.O. Box MS 3102

Lubbock, TX 79409-3102
Zuliang Shen

National University of Singapore

Institute of Systems

Heng Mui Keng Terrace

Kent Ridge 0511

Singapore

Takanori Shibata

MITI

Biorobotics Division

Mechanical Engineering Laboratory

Namiki 1-2

Tsukuba-shi Ibaraki 305

Japan

Divyendu Sinha

CUNY/College of Staten Island

Computer Science Department

2800 Victory B $\mathrm{V}$ vd.

Staten Island, NY 10314-6609

Purnendu Sinha

Stevens Institute of Technology

Electrical Engineering and Computer

Science Department

Castle Point Station

Hoboken, NJ 07030

Dandina H. Rao

807-107, Cumberland

Saskatoon SK S7N OW0

Canada

xiv 